\title{
Sinergin educación, prevención, promoción de salud comunitaria desde el paradigma de la sociocultura
}

\author{
José Alfredo Villarreal Valera*
}

Recibido en enero 23 de 2019, aceptado en octubre 1 de 2019

\section{Citar este artículo así:}

Villarreal JA. Sinergia educación, prevención, promoción de salud comunitaria desde el paradigma de la sociocultura. Hacia. Promoc. Salud. 2020; 25 (2): 39-53 DOI: 10.17151/hpsal.2020.25.2.7

Objetivo: Analizar la sinergia de la educación, prevención y promoción de la salud concebida como una construcción sociocultural mediada por las prácticas culturales que desarrollan las personas de una comunidad cubana durante los años 2013-2017. Materiales y Métodos: Estudio de caso único que combina las perspectivas cuantitativa y cualitativa de investigación, con el empleo de la observación participante, cuestionarios, entrevistas en profundidad en el escenario comunitario y la consulta a expertos, además de los procedimientos teóricos analítico-sintético, así como la inducción y deducción. Resultados: Los resultados revelan la existencia de prácticas culturales comunitarias relacionadas con la salud, en las que se produce una simbiosis de la educación, prevención y promoción de salud como elementos esenciales del proceso salud-enfermedad. Conclusiones: El contexto comunitario en que se desarrolla el proceso salud-enfermedad está mediado por prácticas culturales, lo que propicia la concepción de la salud como una construcción sociocultural inherente a la actividad humana, la cual debe garantizarse mediante la educación, prevención y promoción de salud concebidas como todo un sistema integrado.

\section{Palabras clave}

Educación en salud, prevención, promoción de la salud, salud pública, factores sociológicos (fuente: DeCS, BIREME).

\footnotetext{
*Licenciado en Filosofía, Universidad de Granma, Bayamo, Cuba. Autor para correspondencia. Correo electrónico: jvillarrealv@udg.co.cu (D_orcid.org/0000-0002-3757-293X. Google
} 


\title{
Synergy between education, prevention, promotion of community health from the sociocultural paradigm
}

\begin{abstract}
Objective: To analyze the synergy between education, prevention and promotion of health conceived as a sociocultural construction mediated by the cultural practices developed by people of a Cuban community during the years 2013-2017. Materials and Methods: Single case study that combines the quantitative and qualitative perspectives of research with the use of participant observation, questionnaires, indepth interviews in the community setting and the consultation to experts, besides the analytic-synthetic theoretical procedures, as well as induction and deduction. Results: The results reveal the existence of community cultural practices related to health in which a symbiosis between education, prevention and promotion of health as essential elements of the health-disease process is produced. Conclusions: The community context in which the health-disease process takes place is mediated by cultural practices which favors the conception of health as an sociocultural construction inherent to the human activity, which must be guaranteed through education, prevention and promotion of health conceived as an entire integrated system.
\end{abstract}

\section{Key words}

Health education, prevention, health promotion, public health, sociological factors (Source: MeSH, NLM).

\section{Sinergin educação, prevenção, promoção da saúde comunitorio desde o paradigma socio-cultural}

\section{Resumo}

Objetivo: Analisar a sinergia da educação, prevenção e promoção da saúde concebida como uma construção sociocultural mediada pelas práticas culturais que desenvolvem as pessoas duma comunidade cubana durante os anos 2013-2017. Materiais e Métodos: Estudo de caso único que mistura as perspectivas quantitativa e qualitativa de pesquisa, com o emprego da observação participante, questionários, entrevistas em profundidade no cenário comunitário e a consulta a expertos, além dos procedimentos teóricos analítico-sintético, assim como a indução e dedução. Resultados: Os resultados revelam a existência de práticas culturais comunitárias relacionadas com a saúde, nas que se produze uma simbioses da educação, prevenção e promoção de saúde como elementos essenciais do processo saúde- doença. Conclusões: O contexto comunitário no que se desenvolve o processo saúde-doença está mediado por práticas culturais, o que propicia a concepção da saúde como uma construção sociocultural inerente à atividade humana, a qual deve garantir-se mediante a educação, prevenção e promoção de saúde concebidas como um sistema integrado.

\section{Palavras Chave}

Educação em saúde, prevenção, promoção da saúde, saúde pública, fatores sociológicos (Fonte: DeCS, BIREME). 


\section{Introducción}

Proteger, conservar, mejorar la salud, prolongar y asegurar la calidad de vida de las poblaciones mediante la educación, la prevención de la enfermedad, la promoción de la salud, y otras formas de intervención sanitaria, constituyen objetivos esenciales de los sistemas sociales en los que el ser humano sea lo más importante.

Es necesario tener presente que la salud existe teóricamente en unidad y contraposición con la enfermedad, o sea, como par de categorías dialécticas que se excluyen y a la vez se presuponen entre sí. De ahí la validez e importancia de esta afirmación: hay que trasladar el énfasis de lo curativo a lo preventivo, centrarse en estrategias de promoción de salud y prevención de las enfermedades, lo cual está presente en el discurso, pero sigue deficiente en la práctica social (1).

Los estudios teóricos relacionados con la educación, prevención y promoción de la salud, desde la perspectiva de la sociocultura, revisten hoy gran importancia en tanto la cultura y la salud constituyen fenómenos sociales intrínsecos de la vida cotidiana, son un aspecto esencial de la vida social (2).

En las condiciones del convulso siglo XXI la salud se legitima como uno de los bienes más preciados, condición indispensable para garantizar el proceso de producción y reproducción social, el desempeño de roles y la realización de la vida cotidiana, como elementos esenciales para lograr una adecuada calidad de vida.

El estudio de los problemas de salud logra integralidad, cuando parte de su consideración como problema social. La necesidad de estos estudios se justifica en la medida en que se comprenda que la salud humana es un fenómeno dinámico que se desarrolla en un contexto cultural, político, social y económico determinado y en total dependencia del desarrollo social. Por tanto, la salud de la población no puede ser desvinculada del desarrollo y progreso social, su campo de estudio y su práctica se imbricará tanto con el área del saber de las ciencias médicas y de la salud como con las diferentes disciplinas sociales.

$\mathrm{Si}$ la salud depende de comportamientos individuales, determinantes sociales, del desarrollo y la justicia alcanzada en el contexto social donde el hombre vive, promover salud es responsabilidad de todos los sectores de la sociedad y dependerá en última instancia de la base económica y la equidad de su distribución. Los sistemas y servicios de salud deben priorizar las acciones educativas, preventivas y de promoción de salud con accesibilidad a todos los grupos poblacionales.

Es por lo anterior que los problemas de salud son abordados en los diferentes campos de las ciencias sociales, entre ellos la sociología $(3,4)$ y la antropología. Clásicos de la sociología como Émile Durkheim, con su concepción acerca de hecho social (5) reconocido como punto de partida para la investigación sociológica en salud que permite la comprensión de los problemas de salud en su dimensión de problema social en general; Talcott Parsons, quien fue el primero en ofrecer un concepto de salud plenamente estructurado sobre términos puramente sociológicos y Pierre Bourdieu con sus aportaciones de los conceptos de habitus, campo y capital simbólico, incursionan desde sus perspectivas teóricas sociológicas en problemas de la salud $(6,7)$ que se sumen como referentes en los que se basa el artículo. Más tarde se van incorporando la psicología social y la economía, la demografía, la historia y la geografía médica.

Otros investigadores sociales de reconocido prestigio internacional como Lévi-Strauss, Malinowski y el propio Durkheim evidenciaron la influencia de los aspectos socio-culturales en las relaciones familiares y en determinados problemas de salud.

Dado que la salud depende directamente del fenómeno social en que está inmersa, entonces un 
análisis de la situación de salud no puede obviar sus determinantes sociales: pobreza, globalización, inequidad, migración, deuda y privatización, alimentación, salario, vivienda, educación, medio ambiente, trabajo, estrés, violencia y adicciones (10), entre otros, cuyo desarrollo está condicionado por las relaciones entre las prácticas culturales de salud y la estructura social.

Lo anterior significa que la definición social de la salud incluye desde el estado de desarrollo del país y el rol social que cada individuo debe cumplir en un campo determinado (de acuerdo con Pierre Bourdieu: espacio de relaciones entre diferentes agentes que ejercen una misma actividad, por ejemplo, artístico, académico, religioso, deportivo, escolar, científico, etc., en pugna por alcanzar un capital simbólico para legitimarse; prácticas de clase), hasta las prácticas culturales que les son intrínsecas. Estas prácticas son entendidas como tipo específico de prácticas sociales o según las define el sociólogo francés, como sistema de disposiciones fuertemente estructurada en las relaciones sociales, tipo específico de prácticas sociales y de significados, casi inabarcables en su totalidad, acciones en busca de objetivos vitales, como la salud, en distintos tiempos y escenarios sociales, que incluyen cultura, hábitos, costumbres, creencias, conductas de salud, etc.

Una mirada de este asunto desde el paradigma de la sociocultura (11) requiere del análisis de las concepciones mágico-religiosas, costumbres, conductas, hábitos, estilo de vida, calidad de vida, modo de vida, tradiciones, mediaciones, mentalidades, memoria común, percepciones, representaciones, significaciones e imaginario que sobre la salud, la enfermedad y la atención médica tiene la población, el grado de satisfacción con los servicios de salud, las características de la relación médico paciente, deseos, preferencias, expectativas, oportunidades, acceso en términos culturales, información y evaluación de los servicios, así como el impacto en la población de las campañas de educación, prevención y promoción de salud, entre otros.
A su vez, este enfoque sociológico supone el abordaje de la salud en su interrelación con las prácticas culturales (12) y las prácticas médicas enmarcadas en un proceso cultural diferencial en los distintos niveles de la organización social, partiendo de que el comportamiento humano está determinado por grupos y clases sociales y por la interacción que tiene lugar entre ellos, donde la responsabilidad máxima descansa en la comunidad misma, dotada de patrones culturales específicos, que es además el espacio donde interactúan lo biológico, lo social y lo cultural, donde existen los problemas de salud.

Las ideas iniciales anteriores conducen a la definición del objetivo principal del presente artículo de investigación científica, el cual consiste en analizar la sinergia de la educación, prevención y promoción de la salud concebida como una construcción sociocultural mediada por las prácticas culturales que desarrollan las personas, por lo que resultan pertinentes las reflexiones y referentes sociológicas mencionadas, al tratar de explicar el binomio salud-enfermedad como un sistema que se legitima como institución social y el condicionamiento cultural de la salud como problema social. Tales reflexiones aportan postulados epistémicos-conceptuales y metodológicos esenciales para comprender este tema.

\section{Métodos}

En la investigación se desarrolló una estrategia metodológica a partir de un diseño global de caso único, que al combinar las perspectivas cuantitativas y cualitativas permitió el empleo de diversos métodos y técnicas para la recogida de información, con predominio de la observación participante, cuestionarios, entrevistas en profundidad y la consulta a expertos, se emplean los procedimientos teóricos analítico-sintético, así como la inducción y deducción. Dicha estrategia incluye, además, la triangulación de tipo teórica y metodológica combina el enfoque cualitativo 
con el cuantitativo en un estudio de caso, lo que permite estudiar y reconocer que la salud comunitaria es un fenómeno tanto objetivo como subjetivo (8) y posibilita organizar el pensamiento con un enfoque integrador de esta, basado en la concepción sociosemiótica de la cultura.

La metodología empleada orienta el trabajo que en el terreno empírico ha realizado el autor, lo que permite demostrar su idea central cuya esencia se expresa mediante el conocimiento de la sinergia educación, prevención, promoción de la salud en la comunidad objeto de estudio. Este se desarrolló en el período 2013-2017, en el Consejo Popular "El Valle, Manopla, La Unión" (17825 pobladores como unidad de análisis) de la ciudad de Bayamo, provincia Granma, Cuba, pues el mismo está compuesto por tres comunidades pequeñas, cercanas, cuya delimitación es puramente oficial, pues sus orígenes, proceso de formación y rasgos son comunes, entendida como comunidad de vecinos, por lo que los investigadores lo considera como una totalidad única, de forma global, como una única unidad de análisis cuyo marco teórico que subyace al estudio es por sí mismo de carácter global (9). No obstante, se seleccionó la unidad de observación o muestra compuesta por 1300 habitantes.

Los métodos y técnicas empleadas permitieron a los investigadores evaluar el comportamiento de las prácticas culturales que, relacionadas con la salud desde la educación, prevención y promoción, se desarrollan por parte de los comunitarios, para lo cual se basaron en las variables preferencias en el acceso a los servicios de salud o empleo de prácticas de la medicina alternativa, empleos de sustancias tóxicas como mecanismos estabilizadores de la dinámica salud-enfermedad, estilo de vida, hábitos higiénico-sanitarios y chequeo médico preventivo como prácticas para cuidar o preservar la salud.
El estudio de casos transitó por distintas fases o etapas para la contrastación de las ideas teóricas que defendemos: fase de exploración-diagnóstica, fase de aplicación de métodos, técnicas e instrumentos y fase de análisis y sistematización de la información.

Para la selección del caso se tuvieron en cuenta tres criterios: el Consejo Popular seleccionado como caso objeto de estudio es uno de los más poblados; el que posee las peores condiciones socioeconómicas y es un espacio social donde se manifiestan fuertes tradiciones culturales, heterogeneidad en prácticas de cultos religiosos, alta presencia de adicciones y hábitos tóxicos, alta prevalencia de enfermedades infectocontagiosas, deficientes hábitos higiénicosanitarios, numerosas conductas antisociales y violentas, así como la existencia significativa de conductas suicidas.

\section{Resultodos}

Los métodos y técnicas empleadas permitieron a los investigadores evaluar el comportamiento de las prácticas culturales que, relacionadas con la salud desde la educación, prevención y promoción, se desarrollan por parte de los comunitarios.

La investigación evidencia resultados expresados en los datos ofrecidos por la población tomada como muestra a conveniencia del investigador (Tablas 1-4) en los que se puede concluir que salud es un concepto de máxima generalidad que incluye la sinergia entre los aspectos educativo, preventivo, y de promoción, así como las pautas, patrones y modos conductuales (prácticas culturales de la comunidad relacionadas con la salud) asimilados e incorporados por los comunitarios en el transcurso de la vida pre y post natal para garantizar un adecuado equilibrio metabólico y socio-psicológico con el medio natural y social. 
Tabla 1. Preferencias en el acceso a los servicios de salud o empleo de prácticas de la medicina alternativa en población Consejo Popular Valle-Manopla-La Unión, Bayamo (2013-2017).

\begin{tabular}{lcc}
\hline Variable acceso a los servicios de salud o a la medicina alternativa & Cantidad & $\%$ \\
\hline Consultar al médico & 24 & 48 \\
\hline Acceder a curanderos o yerbateros & 30 & 60 \\
\hline Sobarse & 45 & 90 \\
\hline Santiguarse & 38 & 76 \\
\hline Dejarlo en manos de dios & 20 & 40 \\
\hline Automedicarse & 38 & 76 \\
\hline Combinar las anteriores & 40 & 80 \\
\hline No hacer nada, esperar & 22 & 44 \\
\hline
\end{tabular}

Fuente: Elaboración propia a partir de los datos aportados por la observación participante y las entrevistas, cuestionarios y otras técnicas aplicadas en el estudio de caso.

Los datos contenidos en la tabla 1 permiten advertir que las prácticas de salud están mediadas por prácticas culturales, por lo que la tendencia general mayoritaria de la población es acceder a la medicina alternativa tradicional, de origen no científico, sino, asociado con las tradiciones y creencias sobrenaturales, frente a la medicina científica, o cuando menos, combinar ambas opciones. Claramente dichas prácticas requieren de un sistemático accionar educativo en salud que se proponga formar conocimientos sobre la importancia y necesidad de acudir a la atención médica oficial.

Tabla 2. Empleo de sustancias tóxicas en población Consejo Popular Valle-Manopla-La Unión, Bayamo (2013-2017).

\begin{tabular}{lcc}
\hline \multicolumn{1}{c}{ Variable empleo de sustancias tóxicas } & Cantidad & $\%$ \\
\hline Consumo de alcohol & 35 & 70 \\
\hline Tabaquismo & 40 & 80 \\
\hline Drogas & 8 & 16 \\
\hline Relajantes o sedantes, estimulantes, tranquilizantes & 47 & 94 \\
\hline
\end{tabular}

Fuente: Elaboración propia a partir de los datos aportados por la observación participante y las entrevistas, cuestionarios y otras técnicas aplicadas en el estudio de caso.

La tabla 2 da fe de lo alarmante de la existencia de prácticas culturales que se manifiestan en las adicciones y hábitos de consumo de sustancias tóxicas o estupefacientes en índices elevados de la población objeto de estudio, lo que incide negativamente en la salud comunitaria y de manera significativa es una de las causas fundamentales de la alta prevalencia de enfermedades infectocontagiosas en la comunidad. Por otro lado, se evidencia la necesidad de intensificar las acciones de prevención de salud enfocadas a revelar los riesgos que acompañan a esos hábitos. 
Tabla 3. Estilo de vida en población Consejo Popular Valle-Manopla-La Unión, Bayamo (2013-2017).

\begin{tabular}{lcc}
\hline \multicolumn{1}{c}{ Variable estilo de vida de la población } & Cantidad & $\%$ \\
\hline Inadecuada alimentación & 38 & 76 \\
\hline Estrés & 46 & 92 \\
\hline Inadecuado empleo del tiempo libre & 15 & 30 \\
\hline No realizar actividades de recreación & 44 & 88 \\
\hline No realizar ejercicios físicos & 45 & 90 \\
\hline Práctica de la promiscuidad & 5 & 10 \\
\hline Obesidad & 23 & 46 \\
\hline Sedentarismo & 23 & 46 \\
\hline $\begin{array}{l}\text { Soledad (ausencia de acompañamiento familiar, de pareja, de } \\
\text { amistades) }\end{array}$ & 12 & 24 \\
\hline
\end{tabular}

Fuente: Elaboración propia a partir de los datos aportados por la observación participante y las entrevistas, cuestionarios y otras técnicas aplicadas en el estudio de caso.

Tabla 4. Hábitos higiénico-sanitarios y chequeo médico preventivo en población Consejo Popular ValleManopla-La Unión, Bayamo (2013-2017).

\begin{tabular}{lcc}
\hline \multicolumn{1}{c}{ Variable hábitos higiénico-sanitarios y chequeo preventivo } & Cantidad & $\%$ \\
\hline Frecuente lavado de manos & 22 & 44 \\
\hline Baño diario & 48 & 96 \\
\hline Frecuente cambio de ropas & 38 & 76 \\
\hline Cepillado con la frecuencia aconsejada & 6 & 12 \\
\hline Chequeo periódico & 2 & 2 \\
\hline Chequeo ocasional & 5 & 10 \\
\hline Solamente cuando se siente mal & 15 & 30 \\
\hline Casi nunca & 6 & 12 \\
\hline Nunca & 22 & 44 \\
\hline
\end{tabular}

Fuente: Elaboración propia a partir de los datos aportados por la observación participante y las entrevistas, cuestionarios y otras técnicas aplicadas en el estudio de caso.

Las tablas 3 y 4 muestran resultados que se interconectan desde lo educativo y la promoción, en tanto, se trata de prácticas culturales que articulan directamente con las determinantes del estado de salud como el estilo de vida y los hábitos higiénico-sanitarios.

Los resultados explicitados en las argumentaciones teóricas, en las valoraciones cualitativas y en los datos que ofrecen las tablas, revelan la existencia de prácticas culturales comunitarias relacionadas con la salud, en las que se requiere el importante papel de la sinergia de la educación, prevención y promoción de salud como elementos esenciales del proceso salud-enfermedad, la que cuando está ausente o es insuficiente en su sistematicidad, como en el caso objeto de estudio, provoca serios daños sociales y a la salud. Por ejemplo, alta presencia (94\%) de adicciones y hábitos tóxicos, alta prevalencia (60\%) de enfermedades infectocontagiosas, deficiente percepción de riesgos por parte del $70 \%$ de la población, deficientes hábitos higiénico-sanitarios en el 70\% de la población, numerosas conductas antisociales y violentas, así como la existencia significativa de conductas suicidas (20\%), alteraciones del sistema 
nervioso, trastornos psicológicos, hipoglucemias, estados de hipertensión e hipotensión arterial, disfunciones sexuales, todas entre 50 y $60 \%$, entre otras.

Por otro lado, la investigación permitió obtener otros resultados de tipo sociocultural que están vinculados con tales prácticas culturales y son factores causales de las conductas anómalas de la comunidad que atentan contra la sinergia educación, promoción y prevención de salud. Entre ellas:

- La comunidad objeto de estudio se originó a partir del éxodo de familias procedentes de zonas rurales por lo que existe un arraigo a costumbres, tradiciones, estilos de vida, comportamientos, hábitos higiénicos y de consumo, símbolos compartidos, formas de comunicarse, propios de esos territorios de raíces campesinas.

- La comunidad no posee alcantarillado, ni redes viales pavimentadas. La disposición de residuales líquidos y sólidos es deficiente, también lo es la recogida de desechos, lo que da lugar a la existencia de microvertederos.

- Es frecuente la práctica del fecalismo al aire libre, constatándose, además, mediante la observación participante, las dificultades con la higiene de los alimentos en varias instituciones sociales.

- En general el abasto de agua es muy deficitario, al no existir redes del acueducto en la zona y tener los moradores que abastecerse de un servicio popular de abasto de agua a partir de la compra de barriles del preciado líquido, lo que reviste especial importancia higiénica epidemiológica, pues en muchas ocasiones esto es fuente de contaminación.

- El índice vectorial en la comunidad fue de 2,6 con 53 focos de mosquitos en 2013; ascendiendo a 69 en 2015 y 80 en 2017.

- Se registran en la comunidad altos índices de tabaquismo (80\%) y 1399 casos $(70 \%)$ de alcoholismo, mayoritariamente jóvenes del género masculino y de bajo nivel escolar. En su representación social acerca de este asunto consideran que tal práctica es normal, que no constituye un problema como tal y que no les afecta la salud, sino, todo lo contrario, se sienten más fuertes y vigorosos, más seguros porque "con el alcohol no hay mal que entre".

- Las personas que habitan este territorio poseen bajo nivel escolar, con predominio del nivel primario, practican la crianza de animales domésticos, por ejemplo, la crianza y cuido de caballos para el tiro de coches y de animales de corral dentro de las casas.

- Existencia de muchas viviendas con condiciones constructivas, ambientales y espaciales desfavorables con altos índices de hacinamiento.

- Las principales formas de sostén económico son, en parte, el empleo laboral y en mayor medida la actividad económica informal, consistente fundamentalmente en revender artículos industriales y textiles adquiridos en la red de comercio estatal y la venta de carne de cerdo y otros productos alimenticios.

- Los hogares se constituyen como familias nucleares, pero generalmente devienen en familias extensas de tipo monoparental debido a la inestabilidad de estas y a un alto índice de divorcialidad.

- Los comunitarios poseen maneras específicas de representarse y referirse a las enfermedades, asociándolas al mal de ojos, la brujería, la envidia y en consecuencia tienen preferencias por el uso de medicina alternativa el $70 \%$, fundamentalmente el uso de plantas. Es común en el $80 \%$ de la población la percepción y la práctica de atender la salud mediante la labor de curanderos, sobadores y espiritistas, así como de la automedicación.

\section{Discusión}

A diferencia del enfoque de la educación, prevención y promoción de salud expuesto en este artículo a manera de hallazgo desde la sociocultura 
y de la comprensión sociosemiótica de esa tríada, la salud por lo general o casi siempre ha sido enfocada en el campo de las ciencias médicas por diferentes autores desde las tendencias del pensamiento médico individual biologicista o en ciertas ocasiones desde lo social, permeado por limitaciones teórico-conceptuales; limitándose a enunciar en diversas investigaciones médicas y publicaciones, los factores que constituyen referentes de riesgo para la misma.

Esto significa que al enfocar la salud como fenómeno social los investigadores y autores en dicho campo, centran su atención en los factores de riesgo de todo tipo, incluyendo los biológicos, económicos, medioambientales y otros, mientras que el sociocultural tan solo es mencionado como uno de los determinantes sociales, sin embargo; si observaran las características y la realidad de las comunidades latinoamericanas, no es difícil comprender que en su esencia las prácticas socioculturales relacionadas con el proceso saludenfermedad, deben estar basadas en el principio y la concepción de la salud como construcción cultural.

Algunos autores refieren que la promoción de salud constituye una estrategia que permite recuperar la importancia del entorno social en el desarrollo de la salud propiciando cambios en los estilos de vida de las personas hacia una vida sana. Por ejemplo, Henry E. Sigerist expresaba "la salud se promueve cuando se facilita un nivel de vida decente, buenas condiciones de trabajo, educación, cultura física y medios de descanso y recreación" (13). Sin embargo, en esa generalidad expresada queda excluida la actuación (las prácticas culturales) a lo interno y la responsabilidad individual y colectiva de las personas con respecto a su salud, pues al parecer es desde lo externo (el otro) quien tiene la obligación de facilitarle las condiciones necesarias de salud.

En 1986 auspiciado por la Organización Mundial de la Salud (OMS), Salud y Bienestar del Canadá y la Asociación Canadiense de Salud Pública se organizó la primera Conferencia Internacional sobre promoción de salud conocida como Carta de Ottawa en la que se plantea: las condiciones fundamentales y los recursos para la salud son la paz, la vivienda, la educación, la alimentación, el ingreso, el ecosistema estable, la conservación de los recursos, la justicia social y la equidad. Queda expresado que: la promoción de salud funciona por medio de la acción comunitaria concreta y efectiva para establecer prioridades, tomar decisiones, planear estrategias y desarrollarlas para obtener mejor salud.

Las estrategias deben cubrir un amplio rango de determinantes de la salud: 1. ingreso y posición social, 2. educación, 3. entorno físico, 4. empleo y condiciones de trabajo, 5. características biológicas y condición genética, 6 . desarrollo del niño sano, 7. redes de apoyo social, 8. servicios de salud. La esencia misma de este proceso es darle el poder a la comunidad, rescatar el control y propiedad de su propio comportamiento y destino, aunque se puede percibir la ausencia del factor sociocultural entre los determinantes de salud.

Por su parte, la prevención de la salud es la protección de las amenazas ambientales a la salud y la posibilidad de eliminar enfermedades evitables. De esta definición se deduce, que la prevención de la salud es una de las tareas principales de la salud pública y la medicina, pero contenida en sí misma en la promoción de salud. Con la combinación de estos factores y el reconocimiento de su influencia sobre la salud, nace la idea de que no es suficiente con la asistencia sanitaria para atender al estado de salud de la población. Así, y desde la Conferencia Internacional sobre Promoción de Salud, se comienzan a implementar programas y políticas de salud que atienden a las áreas de actuación en promoción de la salud establecidas en la Carta de Ottawa (22). Estas áreas serían:

- Construir políticas saludables: contribuir a la salud mediante la adopción de medidas políticas que favorezcan la salud en aquellos 
sectores no directamente implicados en la misma.

- Crear ambientes que favorezcan la salud: fomentar el apoyo para protegernos los unos a los otros, así como a nuestras comunidades y a nuestro medio natural, contribuyendo a la creación de ambientes y entornos tanto físicos, como psicológicos y sociales que conduzcan al bienestar y productividad de la comunidad y a controlar o eliminar factores de riesgo para la salud; su propósito fundamental es crear o sostener ambientes y procesos favorables al aprendizaje de habilidades para la vida.

Desarrollar habilidades personales: que la promoción de la salud favorezca el desarrollo personal y social mediante la información, la educación y el desarrollo de aptitudes indispensables para la vida. Así se aumenta el "empoderamiento" de la población, lo que significa dar herramientas a la comunidad para que ejerza un mayor control sobre su 46 propia salud y sobre el medio ambiente y para que opte por todo lo que le propicie salud.

- Reforzar la acción comunitaria: como una práctica efectiva en la fijación de prioridades, en la toma de decisiones y en la elaboración y puesta en marcha de acciones de planificación para alcanzar un mejor nivel de salud. En el caso de los escolares seria mejorar la participación entre la escuela y la comunidad, involucrando madres y padres de familia, el sector público y privado y la sociedad en general en torno a su bienestar.

- Reorientar los servicios de salud: Como un trabajo de toda la sociedad para la consecución de un sistema de protección de la salud que propicie que se trascienda la prestación de servicios médicos y clínicos y que los servicios de salud se orienten a las necesidades culturales de los individuos y favorezcan la necesidad colectiva de una vida más sana.

Coincidiendo con lo que se expone por estas instituciones y documentos, el proceso saludenfermedad hay que analizarlo y atenderlo en su integralidad; sin embargo, es evidente que se centran en cuestiones biomédicas, desconociendo su interconexión con lo cultural que forma parte y en cierta forma regula la conducta de salud de las personas. Por otra parte, todas estas áreas se relacionan entre sí a través de las políticas de salud como interconexión del resto de factores, pues sin ellas es difícil que un Estado logre obtener beneficios en salud. Entre esos factores se encuentran: establecer políticas; crear un entorno favorable; reforzar la acción comunitaria; reorientar los servicios de salud; desarrollar aptitudes y recursos.

Según la Carta de Ottawa, la promoción de la salud consiste en proporcionar a los pueblos los medios necesarios para mejorar su salud y ejercer un mayor control sobre la misma. Para alcanzar un estado adecuado de bienestar físico, mental y social un individuo o grupo debe ser capaz de identificar y realizar sus aspiraciones, de satisfacer sus necesidades y de cambiar o adaptarse al medio ambiente. Y para que esto sea posible, es necesario que se cumplan una serie de principios clave para la promoción de la salud y que son:

- Implicar a la población en su conjunto y en el contexto de su vida diaria, en lugar de dirigirse a grupos de población con riesgo de enfermedades específicas.

Centrarse en la acción sobre las causas o determinantes de la salud para asegurar que el ambiente que está más allá del control de los individuos sea favorable a la salud.

- Combinar métodos o planteamientos diversos pero complementarios, incluyendo comunicación, educación, legislación, medidas fiscales, cambio organizativo y desarrollo comunitario.

- Aspirar a la participación efectiva de la población, favoreciendo la autoayuda y animando a las personas a encontrar su manera de promocionar la salud en sus comunidades.

Aunque la promoción de la salud es básicamente una actividad del ámbito de la salud y del ámbito social, y no un servicio médico, los profesionales sanitarios, especialmente los de atención 
comunitaria, desempeñan un importante papel en apoyar y facilitar la promoción de la salud. Esto hace que la promoción de la salud se dirija a la población en su conjunto, y no sólo a grupos de riesgo o personas enfermas, como hasta el momento.

Se establece como objetivo capacitar a la población para que pueda controlar su propia salud y su enfermedad, y sean más autónomos para que no se dependa única y exclusivamente de los servicios profesionales para tratar la salud. Atendiendo a esto, la promoción de la salud surge como una estrategia complementaria que incluye diversas medidas -legislativas, educativas, económicas, etc. - Al depender la promoción de la salud de la participación activa de la población, la educación para la salud es una herramienta muy importante en este proceso, ya que ésta no solo proporciona a las personas la posibilidad de adoptar creencias, hábitos y actitudes saludables, sino que también conlleva una concienciación de la comunidad para conocer los factores que influyen en su salud $\mathrm{y}$ cómo potenciarlos o transformarlos. El saber cómo alcanzar la salud conlleva una intervención de transformación o refuerzo de los factores que influyen en la salud; intervención en gran medida comunitaria, ya que el grado de salud también depende de factores sociales (14).

Cuando se habla de promoción de salud se incluyen acciones orientadas hacia el mejoramiento de las condiciones de vida de todos los individuos de una comunidad. Esto implica vivienda, seguridad alimentaria, empleo, educación, recreación, ecosistemas estables y ambientes sociales saludables. En este campo se conciben acciones de gran arraigo político y su implementación está supeditada a la concertación entre diversas fuerzas sociales, a la acción intersectorial y, ante todo, al desarrollo económico y social de cada país. Se enmarcan aquí políticas como la de los municipios saludables, impulsada desde la misma OMS. El papel del agente de salud en esta perspectiva se concibe más como dinamizador social, una especie de catalizador de acciones de amplia base comunitaria en una concepción intersectorial.

El mayor impacto de una intervención exitosa en este sentido, se observaría sobre las enfermedades infecciosas y parasitarias con marcada mejoría en la supervivencia de la población materno infantil, más que sobre los estilos de vida. Por ello se utiliza este nivel de supervivencia materno-infantil como indicador comparativo del estado de desarrollo de los programas de promoción de salud en los distintos países.

$\mathrm{Si}$ atendemos a los estudios que se han realizado, se observa que las variables identificadas de especial relevancia en los programas de promoción de salud ignoran a los factores culturales. Por lo general coinciden en incluir:

1. Edad: mientras que, en los grupos más jóvenes de 14 a 17 años, aparece una vertiente moral que señala cierta culpabilidad en el binomio salud / enfermedad (Yo no he hecho nada malo para que me pase algo) acentuando, en ese sentido, la idea de inmunidad propia del joven en el caso de la salud, en los grupos de edad mayor se acentúa la idea de salud desde la perspectiva del estado de armonía entre cuerpo y psique (la salud es el conjunto de bienestar físico y mental)

2. Clase social: también se detecta una influencia determinante respecto de la concepción de la salud en relación con el estatus social de pertenencia del joven; así, mientras que los niveles más bajos poseen un nivel muy precario de información (Yo no sé lo que es el VIH) y trasladan la responsabilidad tanto de la enfermedad como de la información al propio sistema social (ellos tienen que informarnos más), en los estatus más altos, con mayor nivel de información, cobran mayor peso agentes definidos socialmente y cercanos al círculo de los individuos. En este sentido se destaca el papel de la familia como muy importante, tanto en la creación de hábitos (formativos) cuanto de dispositivo de información. 
3. Género: entre hombres y mujeres hay una serie de diferencias respecto a su concepción de salud, debido a multitud de factores tanto psicológicos como fisiológicos, $y$, aunque esto no debe condicionar el tratamiento de los datos que puedan necesitarse para un estudio sobre el tema, sí que han de tenerse en cuenta la hora de analizar estas concepciones y los factores que se relaciona con la salud.

A diferencia de tan exiguo análisis, este autor considera incluir variables que sin negar las anteriores, ejercen una influencia directa sobre la salud individual y comunitaria, a saber: prácticas de acceso a los servicios de salud, prácticas higiénico-sanitarias, prácticas alimentarias, consumo de sustancias tóxicas, la automedicación, estilo de vida de la población, tradiciones, gustos y preferencias culturales.

Es conocido el hecho de que el modelo de atención centrado en la patología ha ejercido su hegemonía y se ha perpetuado por el liderazgo y prestigio del médico en relación con el resto del equipo de salud. El individuo enfermo ha sido el objeto del quehacer médico durante muchos años, pero el enfoque preventivo, presupuso un cambio de atención del individuo enfermo al hombre sano y la evaluación de los factores de riesgo en cualquier comunidad; de modo que se puedan planear acciones encaminadas a suprimir la posibilidad de la enfermedad.

Tanto la promoción, como la prevención de la salud descansan en la educación para la salud como una de las vías principales en su desarrollo.

La OMS define la educación para la salud como la disciplina que se ocupa de iniciar, orientar y organizar los procesos que han de promover experiencias educativas, capaces de influir favorablemente en los conocimientos, actitudes y prácticas del individuo y de la comunidad con relación a la salud. Es un campo especial de las ciencias médicas y de la salud pública cuyo objetivo es la transmisión de conocimientos y el desarrollo de una conducta encaminada a la conservación de la salud, del individuo, el colectivo y la sociedad.

En el Diccionario Terminológico Internacional de Educación para la Salud se define como actividad formativa y educativa, dirigida al desarrollo de una actitud consciente y responsable por parte del hombre ante el fomento, conservación y restablecimiento de la salud y la capacidad de rendimiento. Ejerce su influencia sobre los conocimientos, criterios, convicciones, motivos y actitudes del hombre, en relación con la salud y la enfermedad, y es al mismo tiempo un componente de la formación y educación en general y del sistema específico de la protección de la salud en particular.

Sin embargo, no se debe aceptar la responsabilidad única e individual de las personas y comunidades, pues al Estado corresponde una misión muy importante en la educación, prevención y promoción de sus ciudadanos y, por otro lado, el sector salud no puede actuar solo, porque la mayoría de los factores determinantes de la salud están fuera de su competencia. Se requiere de la cooperación multisectorial.

La tarea consiste, en primer término, en el estudio de la salud y las enfermedades como estados de la vida humana de todas las épocas y culturas, teniendo en cuenta plenamente la condición al mismo tiempo biológica, social y personal del hombre. Ello significa analizar las enfermedades como realidades biológicas cambiantes y sometidas a circunstancias ambientales determinadas, como fenómenos sociales condicionados por estructuras socioeconómicas e integrados en patrones socioculturales concretos y como vivencias personales existentes en cada situación histórica.

La prevención de la enfermedad abarca las medidas destinadas no solamente a prevenir la aparición de esta, tales como la reducción de los factores de riesgo, sino también a detener su avance y 
atenuar sus consecuencias una vez establecida. La prevención primaria está dirigida a evitar la aparición inicial de una enfermedad o dolencia. La prevención secundaria y terciaria tienen por objeto detener o retardar la enfermedad ya presente y sus efectos mediante la detección precoz y el tratamiento adecuado o reducir los casos de recidivas y el establecimiento de la cronicidad, por ejemplo, mediante una rehabilitación eficaz.

La prevención de la enfermedad se utiliza a veces como término complementario de la promoción de la salud. Pese a que a menudo se produce una superposición del contenido y de las estrategias, la prevención de la enfermedad se define como una actividad distinta. En este contexto, la prevención de la enfermedad es la acción que normalmente emana del sector sanitario, y que considera a los individuos y las poblaciones como expuestos a factores de riesgo identificables que suelen estar a menudo asociados a diferentes comportamientos de riesgo.

La educación para la salud comprende las oportunidades de aprendizaje creadas conscientemente que suponen una forma de comunicación destinada a mejorar la alfabetización sanitaria, incluida la mejora del conocimiento de la población en relación con la salud y el desarrollo de habilidades personales que conduzcan a la salud individual y de la comunidad. La educación para la salud aborda no solamente la transmisión de información, sino también el fomento de la motivación, las habilidades personales y la autoestima, necesarias para adoptar medidas destinadas a mejorar la salud.

La educación para la salud incluye no sólo la información relativa a las condiciones sociales, económicas y ambientales subyacentes que influyen en la salud, sino también la que se refiere a los factores de riesgo y comportamientos de riesgo, además del uso del sistema de asistencia sanitaria. Es decir, la educación para la salud supone comunicación de información y desarrollo de habilidades personales que demuestren la viabilidad política y las posibilidades organizativas de diversas formas de actuación dirigidas a lograr cambios sociales, económicos y ambientales que favorezcan la salud.

En el pasado, la educación para la salud se empleaba como término que abarcaba una más amplia gama de acciones que incluían la movilización social y la abogacía por la salud. Estos métodos están ahora incluidos en el término promoción de la salud, y lo que aquí se propone es una definición menos extensa de la educación para la salud para distinguir entre estos dos términos.

Juan César García, en su libro Pensamiento social en Salud en América Latina, define a la medicina, como la ciencia y arte de precaver y curar enfermedades del cuerpo humano. En su significado más amplio, medicina es un campo, región o parte de la sociedad constituida por prácticas y saberes que se diferencian de otros que se dan en esa misma sociedad. La forma más simple para diferenciar estas prácticas sería la de señalar su objetivo más obvio: la prevención y curación de la enfermedad y la preservación de la salud.

En este sentido, se comprende la promoción y la prevención desde la autorresponsabilidad y la autodeterminación de las comunidades, con su debida participación en el diseño e implementación de los programas y con una conciencia proactiva de los determinantes sociales, culturales y ambientales de la salud y la enfermedad.

Llama la atención que la OMS ha instado en diferentes reuniones, talleres y conferencias sobre la necesidad de estudiar, recuperar y promover la medicina tradicional como medio para lograr las metas de salud para todos; sobre la necesidad de reorientar los servicios de salud hacia la promoción de la salud y la prevención de la enfermedad; y sobre la prioridad en el desarrollo y aplicación de modelos asistenciales centrados en la familia y la comunidad con el debido fortalecimiento de la participación en salud $(15,16,17)$. 
La familia es el elemento de la estructura de la sociedad responsable de la reproducción biológica y social del ser humano. Constituye la unidad de reproducción y mantenimiento de la especie humana y en ese sentido es el elemento que sintetiza la producción de la salud a escala microsocial. En ella se satisfacen las necesidades que están en la base de la conservación, fomento y recuperación de la salud (18).

\section{Conclusiones}

Es necesario abandonar la concepción de la salud como un concepto abstracto y asumir la concepción objetiva para definirla dentro del contexto histórico de la sociedad y en el momento específico de su desarrollo.

Sí bien la educación, prevención y promoción para la salud constituyen acciones y estrategias que permiten el fomento y desarrollo de la cultura de la salud, ésta no se reduce a ninguna de ellas. Constituye más bien el resultado general, las consecuencias principales de la aplicación de estas estrategias (19). En las sociedades, la medicina se ocupa principalmente de la lucha contra las enfermedades, no obstante, se ha abierto paso el criterio de que la salud humana requiere que la organización de la sociedad dedique atención a prevenir la enfermedad y promover la salud. Esto ha conducido al enfoque de salud de la población donde se abordan un amplio rango de patrones determinantes de la salud y una estrategia diseñada para llegar a toda la población.
Existe una relación dialéctica entre las categorías educación, prevención y promoción de salud, las cuales son de gran valor para el desarrollo de la medicina en la comunidad.

La prevención significa aplicar medidas para evitar enfermedades, mientras que la promoción se refiere a mejorar las condiciones de salud e incluye la prevención. Por tanto, la promoción es más general que la prevención porque abarca no sólo educación para la salud, sino que incluye, además, modificación de costumbres, hábitos y precisa de un movimiento profundo de la comunidad.

Es una tarea eminentemente social, requiere del esfuerzo mancomunado de muchos factores, tales como: el Estado, condiciones de vida, educadores, de los profesionales de la salud y es muy importante la responsabilidad del individuo y de la familia, de cómo la misma valore su salud y participe en su preservación $(20,21)$. Entre todos estos factores existe una interrelación dialéctica, donde cada uno de ellos desempeña un papel imprescindible y sirve de condición y complemento de los demás.

El contexto comunitario en que se desarrolla el proceso salud-enfermedad está mediado por prácticas culturales, lo que propicia la concepción de la salud como una construcción sociocultural inherente a la actividad humana, la cual debe garantizarse mediante la educación, prevención y promoción de salud concebidas como todo un sistema integrado. 


\section{Referencios}

1. García R. El enfoque social como elemento esencial del quehacer por la salud de la población. Revista Cubana de Salud Pública. Revista Cubana de Salud Pública. 2011. 37 (5): p. 9.

2. Durán M. Las perspectivas sociológicas en el estudio de la salud y la enfermedad. Problemas metodológicos en Sociología y Política de Salud. Selección de Lecturas Editorial Félix Varela, La Habana, 2006.

3. Mercer H. La contribución de la sociología a las investigaciones en salud. En: Rojas Ochoa F, Márquez M, compiladores. ALAMES en la Memoria. Selección de lecturas. Editorial Caminos, La Habana, 2009.

4. Rojo N, Y García R. Sociología y Salud. Reflexiones para la Acción. Revista Cubana de Salud Pública. Revista Cubana Salud Pública, 2000. 26 (2).

5. Durkheim, E. Normas para la explicación de hechos sociales. En Bohannan, Paul y Glazer, Mark. Antropología. Lecturas. Editorial Félix Varela, La Habana, 2005.

6. Alexander J. Las teorías sociológicas desde la segunda guerra mundial. Análisis multidimensional. Editorial Gedisa, España, 1995.

7. Villarreal J. Fundamentos de la teoría sociológica general para el estudio de los problemas relacionados con la salud y la enfermedad. Revista Atlante. Cuadernos de Educación y Desarrollo, Universidad de Málaga, España, 2012.

8. Almaráz, M. Salud Comunitaria y Descentralización. Universidad de la República Oriental de Uruguay, 1999.

9. Rodríguez G, Gil J, García E. Metodología de la Investigación Cualitativa. Editorial Ciencias Médicas (ECIMED), La Habana, 2008.

10. Berlinguer G. Determinantes sociales de la enfermedad. Revista Cubana de Salud Pública [Internet]. [citado 19 Ago 2011]; 33(1), 2007.

11. Donati P. Paradigma médico y paradigma sociológico: las bases teóricas de la sociología sanitaria, en Sociología y Política de Salud. Selección de Lecturas Editorial Félix Varela, La Habana, 2006.

12. Basualdo H. Las prácticas culturales en relación a la sanación. monografías.com. (s/f).

13. Henry S. Promoción de la Salud. Lo acuña por primera vez, definiendo las cuatro tareas esenciales de la medicina: promoción de la salud, prevención de la enfermedad, el restablecimiento de los enfermos y la rehabilitación, 1946.

14. Moncada M. Factores Sociales e Indicadores de Salud en el contexto comunitario. Tesis defendida en opción al grado científico de doctor en Ciencias Sociológicas. Santiago de Cuba, 2000.

15. Reyes I, Sanabria G, Medina Z, Báez R. Metodología para la caracterización de la participación comunitaria en salud. Revista Cubana de Salud Pública. 1996; 22(2):75-84.

16. Rodríguez I, Pérez J. Salud y Participación Social Comunitaria En: Revista Espacio Abierto. Cuaderno Venezolano de Sociología. 1996. 5 (3), 372-373.

17. Sanabria G. Participación social en el campo de la salud. Revista Cubana de Salud Pública. Versión Online. 2004. 30 (3).

18. Bartutis M. La familia y su papel en los problemas de promoción de salud y prevención de enfermedades. Proyecto Editorial CENDECSA, Universidad Médica Carlos J. Finlay, Camagüey, Cuba, 1999.

19. Agirre R, Prieto D. La cultura de la salud, Proyecto Editorial CENDECSA, Universidad Médica Carlos J. Finlay, Camagüey, Cuba.

20. Louro I. La familia en la determinación de la salud. Revista Cubana de Salud Pública; 2003. 29(1): 48-51.

21. Rojo N, Carmona A. La familia y el nivel de salud de la comunidad. Aspectos conceptuales y Metodológicos. Revista Cubana de Administración de Salud. (1982). 8(3):224-41.

22. Carta de Ottawa para la promoción en Salud (Departamento de servicios especiales de información del CNICN). Ottawa. Ontario. Canadá, 1986. 